Proceedings of the 42th "Jaszowiec" International School and Conference on the Physics of Semiconductors, Wisła 2013

\title{
Intershell Exchange Interaction in Charged GaAlAs Quantum Dots
}

\author{
M.R. Molas ${ }^{a, b, *}$, A.A.L. Nicolet ${ }^{b}$, M. Potemski $^{b}$ And A. Babiński ${ }^{a}$ \\ ${ }^{a}$ Institute of Experimental Physics, Faculty of Physics, University of Warsaw, Hoża 69, 00-681 Warszawa, Poland \\ ${ }^{b}$ Laboratoire National des Champs Magnétiques Intenses, CNRS-UJF-UPS-INSA \\ 25, av. des Martyrs, 38042 Grenoble, France
}

\begin{abstract}
Optical anisotropy of charged excitons and biexcitons related to the single-particle $s$ - and $p$-shell emission in GaAlAs/AlAs quantum dots is investigated. The polarization-dependence and time-resolved micro-photoluminescence measurements were performed. Cross-correlation measurements were used to identify the ladder of excitonic states and allowed us to show two cascade pathways, including the spin singlet and triplet states of charged excitons and biexcitons. The fine structure of the studied states is described and analysed in terms of electron-electron, hole-hole, and electron-hole exchange interactions.
\end{abstract}

DOI: $10.12693 /$ APhysPolA.124.785

PACS: 78.67.Hc, 71.35.-y, 78.55.Cr, 71.70.Gm

\section{Introduction}

Anisotropic electron-hole (eh) exchange interactions in quantum dots (QDs) are of great interest because of their potential use in information processing applications [1]. Moreover, multiexcitonic complexes in single QDs are also considered to be interesting candidates for multiphoton emitters as they can emit correlated photons depending on the number of excitons they contain. In practice, a three-photon and four-photon cascade have been demonstrated in the case of a triexciton and a quadexciton $[2,3]$. The quadexciton-triexciton cascade recombination process in single QDs with an asymmetric confining potential is equivalent to the biexciton-exciton cascade process. The former process occurs at the first single-particle (SP) excited state - the $p$-shell, and the second at the SP ground state - the $s$-shell [4].

The exchange interaction in charged multiexcitonic complexes between inter-subshell spins becomes more pronounced than the one in the case of neutral multiexcitonic complexes due to the presence of an excess of carriers. As a result, several split emission lines due to the charged excitonic complexes have been observed in the micro-photoluminescence ( $\mu$-PL) spectra [5-7]. In particular, two decay pathways involving a different spin configuration of charged biexciton states in single GaAs QDs have been identified and described [8].

In this study, we show two decay pathways in cascades involving a spin singlet and a spin triplet state of charged excitons and biexcitons. The fine structure of the investigated states is described and analysed in terms of electron-electron (ee), hole-hole (hh), and eh exchange interactions.

\section{Experimental procedure}

The active part of the structure used for the experiments was intentionally designed as a type-II GaAs/AlAs

${ }^{*}$ corresponding author; e-mail: maciej.molas@fuw.edu.pl bilayer structure. Previous research showed the existence of GaAlAs islands which form at the barrier of AlAs with an extremely low surface density of about $10^{6} \mathrm{~cm}^{-2}$ with an estimated lateral diameter of about $50 \mathrm{~nm}$. A spectrally separated set of lines related to the emission from single GaAlAs/AlAs QDs was observed in the $\mu$-PL spectrum in a broad energy range $(1.56-1.68 \mathrm{eV})[9-11]$.

The investigated sample was located on a cold finger of a continuous flow cryostat at liquid helium temperature $(T=4.2 \mathrm{~K})$. A tunable Ti:sapphire laser set at $\lambda=725 \mathrm{~nm}$ was used for excitation. The light was then tightly focused by means of a microscope objective (spot size around $1 \mu \mathrm{m}^{2}$ ). The $\mu$-PL was collected via the same microscope objective, dispersed by a $0.5 \mathrm{~m}$ monochromator and detected by a charge-coupled device camera cooled down with liquid nitrogen [12]. Polarization-resolved experiments were implemented using a motorized rotating half-wave plate combined with a fixed linear polarizer in front of the spectrometer in order to avoid detection artifacts related to the anisotropic response function of the setup. For excitonic lines with small anisotropic splitting, we determined the value of the splitting by fitting a Gaussian curve as a function of the polarization angle and then a cosine square function to obtained energetic position of lines [9].

\section{Results}

The $\mu$-PL spectrum of a typical charged GaAlAs QD as a function of excitation power of the laser is presented in Fig. 1. The investigated dot was excited at an energy below transitions $\left(\Gamma_{\mathrm{GaAs}}-\Gamma_{\mathrm{GaAs}}, X_{\mathrm{AlAs}}^{Z}-\Gamma_{\mathrm{GaAs}}\right.$, $\left.X_{\mathrm{AlAs}}^{X Y}-\Gamma_{\mathrm{GaAs}}\right)$ in the type-II GaAs/AlAs bilayer $[9,11]$. In this so-called quasi-resonant excitation regime, eh pairs are created directly in the QD, conditions which favor neutral charge states [13]. However, additional effects like e.g. doping of the structure or a different lifetime of carriers (electrons and holes) lead to the observation of lines related to charged states.

Identification of the sign of singly charged excitons labelled in Fig. 1 is based on basic arguments related to 


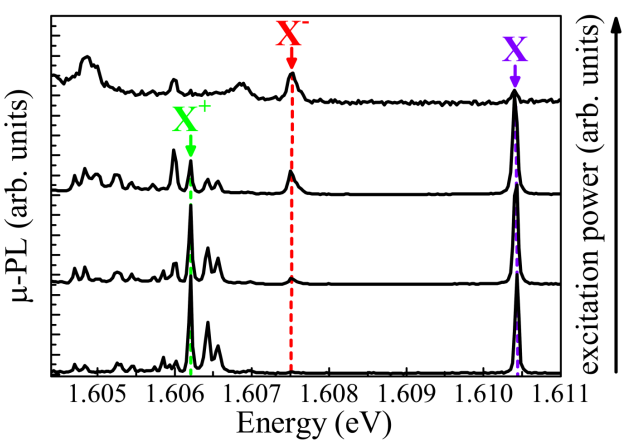

Fig. 1. Excitation-power evolution of the $\mu$-PL spectra from a single GaAlAs/AlAs dot measured at low temperature without polarization resolution. The spectra are rescaled and shifted for clarity purpose.

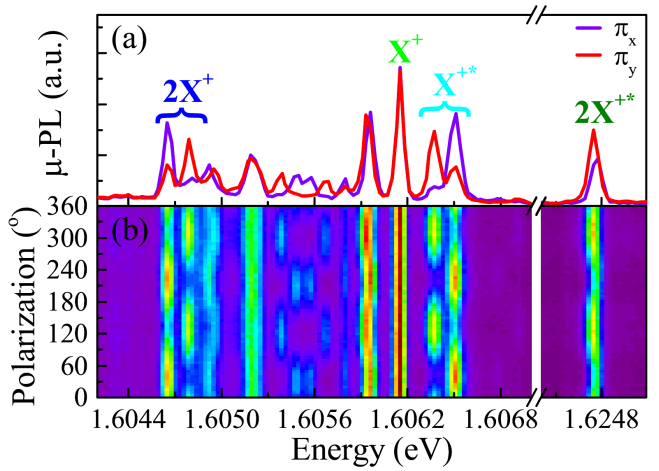

Fig. 2. (a) The $\mu$-PL spectra of a single GaAlAs QD. Polarization axes: $\pi_{x}$ (violet curve) and $\pi_{y}$ (red curve) along crystallographic axes [110] and [110], respectively. (b) Color plot presenting $\mu$-PL spectra of the same QD measured using different linear polarization of detection. The $45^{\circ}$ and $135^{\circ}$ correspond to the crystallographic axes [110] and [110].

the studied structure. In the polarization-resolved $\mu$-PL experiment, no splitting of the $\mathrm{X}^{-}$and $\mathrm{X}^{+}$transitions was detected, as shown in the case of the $\mathrm{X}^{+}$line in Fig. 2. This is expected since the eh exchange interaction influences neither the initial (where the two majority carriers are forming closed shell with $J_{\mathrm{e}}=0$ ) nor the final state (only one carrier left). It has been previously reported that the $\mathrm{X}^{+}$emission is not observed and the $\mathrm{X}^{-}$is clearly observed in non-resonant excitation regime [9], due to the very long lifetime of the indirect excitons at the type-II GaAs/AlAs bilayer [14]. In a quasi-resonant excitation regime, the emission related to the negatively charged exciton $\left(\mathrm{X}^{-}\right)$has also been observed and identified, based on the particular shape of the QD confining potential, significantly deeper for electrons than for holes [11]. However, it is known that nominally undoped GaAs-based structures exhibit a residual $p$-type background doping. We conclude that the line labelled $\mathrm{X}^{+}$in Fig. 1 is related to the spin singlet state of the positively singly charged exciton.

In this paper, we focus on the analysis of the fine structure of the positively charged states related to the $s$ - and $p$-shell emissions. All investigated QDs presented similar emission patterns. Excitonic and biexcitonic transitions split into pairs of linearly polarized lines, exhibiting the same absolute value with opposite signs. For the QD with the spectrum shown in Fig. 2 the exchange interaction splitting of the neutral state equals to $16 \mu \mathrm{eV}$, which is relatively small in comparison with the charged transitions presented in Fig. 2.

The emission lines due to the recombination of positively charged multiexcitons have a more complex fine structure. Figure 2a shows a typical $\mu$-PL spectrum of charged GaAlAs QD as a function of linear polarizations along the principal axes of the QD. The identification of studied lines was done by cross-correlation measurements of all investigated lines [15]. Emission lines $\mathrm{X}^{+}, 2 \mathrm{X}^{+*}$ and $\mathrm{X}^{+*}, 2 \mathrm{X}^{+}$belong to separate cascade pathways. Transitions, $\mathrm{X}^{+*}, 2 \mathrm{X}^{+}$, and $2 \mathrm{X}^{+*}$, split into doublets partially linearly polarized along the same two principal crystallographic axes [110] and [110] (see Fig. 2), which have been identified for the $\mathrm{X}$ emission in [9]. In order to discuss the energy structure of the two cascade pathways involving singlet and triplet states of charged excitons and biexcitons, we use the many-carrier Hamiltonian equation for QDs given in [7]. This equation is used to describe levels splitting by the exchange interaction for the states involved in the investigated cascades. It should be noted that the degeneracy of the $p$-shell is important for the spin states. We assumed that the splitting of $p$ orbitals is larger than the $p_{x}-p_{y}$ hole exchange energy [16].

Theoretical description of the fine structure of investigated states is schematically presented in Fig. 3. We identified two separate cascade pathways of excitonic emissions involving states $\mathrm{X}^{+*}, 2 \mathrm{X}^{+}$(Fig. 3a), and $\mathrm{X}^{+}$, $2 \mathrm{X}^{+*}$ (Fig. $3 \mathrm{~b}$ ) related to the $s$ - and $p$-shell. The fine structure of transitions shown in Fig. 2a, involving the spin triplet state of a charged exciton $\left(\mathrm{X}^{+*}\right)$ and the spin singlet state of a charged biexciton $\left(2 \mathrm{X}^{+}\right)$are related to the $\mathrm{X}^{+*}$ state. The structure of $2 \mathrm{X}^{+}$transition results from the splitting of the final state of an $s$-shell electron $\left(\mathrm{e}_{s}\right)$, an $s$-shell hole $\left(\mathrm{h}_{s}\right)$, and a $p$-shell hole $\left(\mathrm{h}_{p}\right)$. The final state consists of three interacting carriers: $\mathrm{e}_{s} \mathrm{~h}_{s} \mathrm{~h}_{p}$. The two holes form a spin-singlet $\left(J_{\mathrm{h}}=0\right)$ and a spin-triplet $\left(J_{\mathrm{h}}=3\right)$ states split by the hh exchange energy $V_{s, p}^{\mathrm{hh}}$. In addition, the eh exchange interaction splits the triplet states into three doublets separated by $\tilde{V}_{s, s p}^{\mathrm{eh}}=\left(V_{s, s}^{\mathrm{eh}}+V_{s, p}^{\mathrm{eh}}\right) / 2$, which includes the eh exchange energies between respectively holes and the $\mathrm{e}_{s}$ electron. It is known that the hh (ee) isotropic exchange energy is larger than the eh exchange energy by one order of magnitude [5]. In optical transitions, the lowest triplet states $J_{z}=7 / 2$ in which the three particles have parallel spins, is forbidden. Note that in our experiments we cannot identify the $2 \mathrm{X}^{+}$transition which is related to the recombination of the spin singlet state (dashed blue transition presented in Fig. 3a). Opposite to the case of the $2 \mathrm{X}^{+}$emission, the $\mathrm{X}^{+*}$ structure transition results from the splitting of the initial state (no interaction is present in the final state due to $h_{p}$ ). The anisotropic 
(a)

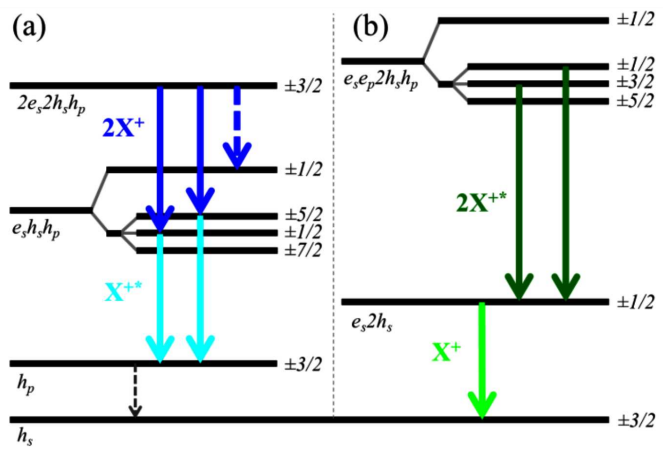

Fig. 3. Theoretical structures (not to scale) of various positively charged transitions in a single QD. The (a) and (b) parts describe two separate cascade pathways. The $\mathrm{e}_{s}\left(\mathrm{~h}_{s}\right)$ and $\mathrm{e}_{p}\left(\mathrm{~h}_{p}\right)$ denote electron (hole) occupying the $s$ - and $p$-shell, respectively. The half-integers are the projections $J_{z}=J_{z}^{\mathrm{e}}+J_{z}^{\mathrm{h}}$ of the total spins.

part of the eh exchange interaction leads to the partial linear polarization of both visible transitions $\left(2 \mathrm{X}^{+}, \mathrm{X}^{+*}\right)$ in this cascade pathway (see Fig. 3a).

In the other cascade pathway presented schematically in Fig. 3b, the fine structure of the triplet spin state of the charged biexciton $\left(2 \mathrm{X}^{+*}\right)$ related to the $p$-shell emission is also analysed. The initial states of the $2 \mathrm{X}^{+*}$ transition, which are responsible for the observed fine structure consist of five carriers: $\mathrm{e}_{s} \mathrm{e}_{p} 2 \mathrm{~h}_{s} \mathrm{~h}_{p}$. The final state of the $2 \mathrm{X}^{+*}$ transition is related to the initial state of the $\mathrm{X}^{+}$emission for which no splitting is observed (see Fig. 2). The obtained fine structure of the $2 \mathrm{X}^{+*}$ state is similar to the fine structure observed in cascade pathway $2 \mathrm{X}^{+} \rightarrow \mathrm{X}^{+*}$. The singlet-triplet energy separations of both states are given by an ee exchange energy $V_{s, p}^{\mathrm{ee}}$. The energy difference between the triplet states are given by an eh exchange energy $\tilde{V}_{s p, p}^{\mathrm{eh}}=\left(V_{s, p}^{\mathrm{eh}}+V_{p, p}^{\mathrm{eh}}\right) / 2$ for the $2 \mathrm{X}^{+*}$ transition.

The analysis of the fine structure of the $\mathrm{X}^{+}$and the $2 \mathrm{X}^{+*}$ transition has been performed for 20 single GaAlAs QDs. The average value for $\tilde{V}_{s, s p}^{\mathrm{eh}}$ is equal to $119 \mu \mathrm{eV}$ and follows the pattern as observed previously for GaAs QDs [8]. In the case of $\tilde{V}_{s p, p}^{\text {eh }}$, the average energy splitting is equal to $63 \mu \mathrm{eV}$ which is nearly $50 \%$ lower than the splitting reported for GaAs dots in former studies [8]. This lower value could be due to different confining potentials, alloy compositions of investigated GaAlAs/AlAs QDs and previously studied GaAs/GaAlAs QDs [8], resulting in differences in ladders of excitonic states.

\section{Conclusions}

We measured 20 single charged GaAlAs/AlAs QDs in order to study the optical anisotropy of charged excitons and biexcitons related to the $s$ - and $p$-shell emission. The attribution of lines was based on cross-correlation measurements between excitonic transitions. The different spin configurations of charged excitons and charged biexcitons were analysed in the light of the polarization-dependence. The observed fine structure splitting (FSS) of the investigated emission lines was described and analysed in terms of the ee, hh, and eh exchange interactions. The obtained average of the FSS values were presented and compared with previously reported results for the GaAs/GaAlAs QDs [8]. We argue that studies on the decrease of the FSS (e.g. by using an external magnetic field) can open the opportunity of the creation of two separate pairs of polarization-entangled photons, which can be of potential use in information processing applications.

\section{Acknowledgments}

The work has been supported by the Foundation for Polish Science International Ph.D. Projects Programme co-financed by the EU European Regional Development Fund and by EuroMagNETII(JRA8) under the EU contract 228043. M.M. kindly acknowledges the National Science Center (doctoral scholarship contract no. UMO-2013/08/T/ST3/00665) for financial support for his Ph.D.

\section{References}

[1] J.-Y. Marzin, J.-M. Gerard, A. Izraël, D. Barrier, G. Bastard, Phys. Rev. Lett. 73, 716 (1994).

[2] J. Persson, T. Aichele, V. Zwiller, L. Samuelson, O. Benson, Phys. Rev. B 69, 233314 (2004).

[3] Y. Arashida, Y. Ogawa, F. Minami, Phys. Rev. B 84, 125309 (2011).

[4] A. Babiński, M. Potemski, S. Raymond, J. Lapointe, Z.R. Wasilewski, Phys. Status Solidi C 3, 3748 (2006).

[5] I.A. Akimov, K.V. Kavokin, A. Hundt, F. Henneberger, Phys. Rev. B 71, 075326 (2005).

[6] R. Heitz, V. Türck, O. Stier, Adv. Solid State Phys. 41, 39 (2001).

[7] E. Poem, J. Shemesh, I. Marderfeld, D. Galushko, N. Akopian, D. Gershoni, B.D. Gerardot, A. Badolato, P.M. Petroff, Phys. Rev. B 76, 235304 (2007).

[8] Y. Arashida, Y. Ogawa, F. Minami, Phys. Rev. B 85, 235318 (2012).

[9] M. Molas, K. Gołasa, B. Piętka, M. Potemski, A. Babiński, Acta Phys. Pol. A 122, 988 (2012).

[10] M.D. Martín, C. Antón, L. Viña, B. Pietka, M. Potemski, Europhys. Lett. 100, 67006 (2012).

[11] B. Piętka, J. Suffczyński, M. Goryca, T. Kazimierczuk, A. Golnik, P. Kossacki, A. Wysmolek, J.A. Gaj, R. Stępniewski, M. Potemski, Phys. Rev. B 87, 035310 (2013).

[12] M. Molas, K. Gołasa, K. Kuldova, J. Borysiuk, A. Babiński, J. Lapointe, Z.R. Wasilewski, J. Appl. Phys. 111, 033510 (2012).

[13] T. Kazimierczuk, J. Suffczyński, A. Golnik, J.A. Gaj, P. Kossacki, P. Wojnar, Phys. Rev. B 79, 153301 (2009).

[14] A. Wysmołek, B. Chwalisz, M. Potemski, R. Stępniewski, A. Babiński, S. Raymond, Acta Phys. Pol. A 106, 367 (2004).

[15] R. Hanbury-Brown, R.Q. Twist, Nature (London) 178, 1447 (1956).

[16] L. He, A. Zunger, Phys. Rev. B 73, 115324 (2006). 